\title{
Three-algebra for supermembrane and two-algebra for superstring
}

\author{
KANGHOON LEE ${ }^{\sharp}$ AND JEONG-HYUCK PARK ${ }^{\dagger}$ \\ \#Department of Physics, Yonsei University, Shinchon-dong, Seodaemun-gu, Seoul 120-749, Korea \\ lkhephya.yonsei.ac.kr \\ ${ }^{\dagger}$ Department of Physics, Sogang University, Shinsu-dong, Mapo-gu, Seoul 121-742, Korea \\ parkesogang.ac.kr
}

\begin{abstract}
While string or Yang-Mills theories are based on Lie algebra or two-algebra structure, recent studies indicate that $\mathcal{M}$-theory may require a one higher, three-algebra structure. Here we construct a covariant action for a supermembrane in eleven dimensions, which is invariant under global supersymmetry, local fermionic symmetry and worldvolume diffeomorphism. Our action is classically on-shell equivalent to the celebrated Bergshoeff-Sezgin-Townsend action. However, the novelty is that we spell the action genuinely in terms of Nambu three-brackets: All the derivatives appear through Nambu brackets and hence it manifests the three-algebra structure. Further the double dimensional reduction of our action gives straightforwardly to a type IIA string action featuring two-algebra. Applying the same method, we also construct a covariant action for type IIB superstring, leading directly to the IKKT matrix model.
\end{abstract}




\section{Contents}

1 Introduction: Two for string and three for $\mathcal{M}$-theory 1

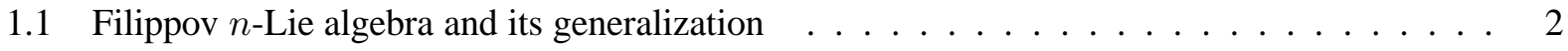

1.2 Reformulation of Nambu-Goto action by Nambu bracket . . . . . . . . . . . . . . 3

2 Three-algebra based action for supermembrane in eleven dimensions 6

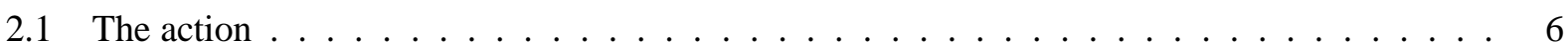

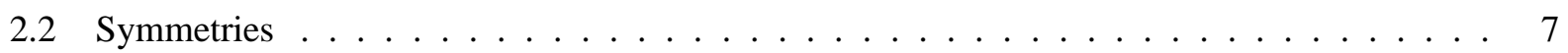

3 Double dimensional reduction to Type IIA superstring theory 9

4 Type IIB superstring theory and IKKT matrix model 10

5 Discussion 12

A Useful Fierz identities in eleven dimensions $\quad 13$

\section{Introduction: Two for string and three for $\mathcal{M}$-theory}

While string and Yang-Mills theories are based on ordinary Lie-algebra or two-algebra structure, recent advances in $\mathcal{M}$-theory by Bagger, Lambert and Gustavsson (BLG) [1,2] suggest that the full description of $\mathcal{M}$-theory may require a generalized Lie-algebra structure: namely three-Lie algebra or shortly threealgebra. In fact, the digits, two and three, appear to have intriguing associations to string and $\mathcal{M}$-theory respectively: First of all, two is the dimension of string worldsheet while three is that of membrane worldvolume. This implies that, after matrix regularization of Poisson bracket structure, IKKT matrix model [3] is a multiple D-instanton description of type IIB superstring via two-algebra, while BFSS matrix model $[4,5]$ is a multiple D0-brane description of eleven-dimensional supermembrane via two-algebra. In other words, the two worldsheet coordinates of a type IIB superstring are traded with matrix indices, while the threedimensional worldvolume of a supermembrane decomposes into ' $1+2$ ', one for the temporal coordinate and two for the matrix indices. Further, two is the codimension of D-branes in each type IIA, IIB superstring theory [6], while three is the codimension of M-branes i.e. M2 and M5. Consequently, through two-algebra interaction as known as Myers effect [7], multiple $\mathrm{D} p$-branes may condense or be polarized into $\mathrm{D}(p+2)$ brane. Similarly, through three-algebra interaction, BLG model equipped with an infinite dimensional gauge group corresponds to a description of the condensation of multiple M2-branes into a single M5-brane [8-20]. Namely, the polarizations of D-branes and M2-branes require two-algebra and three-algebra respectively. 
- 2 for string:

String worldsheet dimension $\Longrightarrow$ IKKT type IIB matrix model 2-algebra structure $\quad \Longrightarrow \quad$ Matrix string/Yang-Mills

Codimension of D-branes $\quad \Longrightarrow \quad$ Myers effect: polarization of $\mathrm{D} p$ into $\mathrm{D}(p+2)$

- 3 for $\mathcal{M}$-theory:

Membrane worldvolume dimension $\quad \Longrightarrow \quad$ BFSS $\mathcal{M}$-theory matrix model

3-algebra structure $\quad \Longrightarrow \quad$ BLG model

Codimension of M2 and M5 $\quad \Longrightarrow \quad$ Condensation of M2s into M5 in BLG model

All the above associations of 2 and 3 to string and $\mathcal{M}$-theory may be naturally understood by a reformulation of the Nambu-Goto action for a $p$-brane. Prior to the explanation, we first review Filippov $n$-Lie algebra and discuss its generalization which is necessary for us later.

\subsection{Filippov $n$-Lie algebra and its generalization}

Filippov introduced $n$-Lie algebra [21] which is a natural generalization of a Lie algebra, defined by $n$ bracket satisfying the totally anti-symmetric property:

$$
\left[X_{1}, \cdots, X_{i}, \cdots, X_{j}, \cdots, X_{n}\right]=-\left[X_{1}, \cdots, X_{j}, \cdots, X_{i}, \cdots, X_{n}\right]
$$

and the Leibniz rule:

$$
\left[X_{1}, \cdots, X_{n-1},\left[Y_{1}, \cdots, Y_{n}\right]\right]=\sum_{j=1}^{n}\left[Y_{1}, \cdots,\left[X_{1}, \cdots, X_{n-1}, Y_{j}\right], \cdots, Y_{n}\right] .
$$

The $n$-Lie algebra can be equipped with an invariant inner product, satisfying the symmetric property,

$$
\langle X, Y\rangle=\langle Y, X\rangle
$$

as well as the invariance under the $n$-bracket transformation,

$$
\left\langle\left[X_{1}, \cdots, X_{n-1}, Y\right], Z\right\rangle+\left\langle Y,\left[X_{1}, \cdots, X_{n-1}, Z\right]\right\rangle=0 .
$$

When $n=2$ the definition reduces to the usual Lie algebra and the inner product can be given by 'Trace'.

Explicitly we may introduce a basis of the $n$-Lie algebra, $T^{a}, a=1,2, \cdots$ and write

$$
\left[T^{a_{1}}, T^{a_{2}}, \cdots, T^{a_{n}}\right]=f^{a_{1} a_{2} \cdots a_{n}} T^{b} .
$$

From (1.1) the structure constant $f^{a_{1} a_{2} \cdots a_{n}}$ is totally anti-symmetric for the upper indices, and the Leibniz rule implies

$$
f^{a_{1} a_{2} \cdots a_{n}} f^{b_{1} b_{2} \cdots b_{n}} a_{n}=\sum_{j=1}^{n} f^{a_{1} a_{2} \cdots a_{n-1} b_{j}} f^{b_{1} \cdots b_{j-1} d b_{j+1} \cdots b_{n}} c
$$


Further the invariant inner product defines a metric $\left\langle T^{a}, T^{b}\right\rangle$ which, along with its inverse, can raise or lower the index $a$. It is worth while to note that in the above expressions all the quantities are assumed to be bosonic. When fermionic variables are present, there must appear extra minus sign if the fermionic quantities are permuted odd times.

One may easily realize the $n$-Lie algebra in terms of Nambu $n$-bracket defined over functional space on an $n$-dimensional manifold [22]:

$$
\begin{aligned}
{\left[X_{1}, X_{2}, \cdots, X_{n}\right] } & \Longleftrightarrow\left\{X_{1}, X_{2}, \cdots, X_{n}\right\}_{\mathrm{N} . \mathrm{B}}:=\frac{1}{\sqrt{\mathcal{G}}} \epsilon^{l_{1} l_{2} \cdots l_{n}} \partial_{l_{1}} X_{1} \partial_{l_{2}} X_{2} \cdots \partial_{l_{n}} X_{n} \\
\langle X, Y\rangle & \Longleftrightarrow \int \mathrm{d}^{n} y \sqrt{\mathcal{G}} X Y
\end{aligned}
$$

In order to ensure the partial integration, either the manifold must be compact or all the functions must vanish on the boundaries of the non-compact manifold. Note that $\mathcal{G}$ corresponds to the determinant of the metric of the manifold, and can be chosen arbitrarily since the properties (1.1), (1.2), (1.4) hold irrespective of the presence of the local factor. In this functional realization of the $n$-Lie algebra, the invariant inner product generalizes:

$$
\langle X, Y, \cdots, Z\rangle \Longleftrightarrow \int \mathrm{d}^{n} y \sqrt{\mathcal{G}} X Y \cdots Z,
$$

such that it satisfies, as a generalization of (1.4),

$$
\sum_{k=1}^{m}\left\langle Y_{1}, Y_{2}, \cdots, Y_{k-1},\left[X_{1}, \cdots, X_{n-1}, Y_{k}\right], Y_{k+1}, \cdots Y_{m}\right\rangle=0
$$

Note that throughout the paper we denote the defining equality by ' $:=$ ' and the on-shell equality as well as gauge fixings by ' $\equiv$ '. For further works on three-algebra see e.g. [23-26].

\subsection{Reformulation of Nambu-Goto action by Nambu bracket}

With an embedding of $(p+1)$-dimensional worldvolume coordinates into $D$-dimensional target spacetime,

$$
X(\xi): \xi^{m} \longrightarrow X^{M}
$$

where $m=0,1, \cdots, p$ and $M=0,1, \cdots, D-1$, the Nambu-Goto action for a $p$-brane reads [27]

$$
S_{\text {N.G. }}=-\int \mathrm{d}^{p+1} \xi \sqrt{-\operatorname{det}\left(\partial_{m} X^{M} \partial_{n} X_{M}\right)} .
$$

Let us decompose, formally, the $p$-brane worldvolume coordinates into two parts:

$$
\left\{\xi^{m}\right\}=\left\{\sigma^{\mu}, \varsigma^{i}\right\}
$$

where $\mu=0,1, \cdots, d-1$ and $i=1, \cdots, \hat{d}$ such that $p+1=d+\hat{d}$. The decomposition is a priori arbitrary for any non-negative integers $d, \hat{d}$. One natural application of the splitting will be the case where 
$p$-brane is extended over two topologically different spaces, e.g. compact and non-compact spaces. With the decomposition above, a square root free reformulation of the Nambu-Goto action was achieved in [13]:

$$
\begin{aligned}
& S=\int \mathrm{d}^{d} \sigma \operatorname{Tr}(\sqrt{-h} L), \quad \operatorname{Tr}:=\int \mathrm{d}^{\hat{d}_{\varsigma}}, \\
& L=-h^{\mu \nu} D_{\mu} X^{M} D_{\nu} X_{M}-\frac{1}{4 \hat{d} !} \omega^{d-1}\left\{X^{M_{1}}, X^{M_{2}}, \cdot \cdot, X^{M_{\hat{d}}}\right\}_{\mathrm{N} . \mathrm{B}}\left\{X_{M_{1}}, X_{M_{2}}, \cdot \cdot, X_{M_{\hat{d}}}\right\}_{\mathrm{N} . \mathrm{B}}+(d-1) \omega,
\end{aligned}
$$

where the action contains three kinds of auxiliary fields: scalar $\omega, d$-dimensional metric $h_{\mu \nu}$ and a gauge connection $A_{\mu}^{i}$ which defines the 'covariant derivative':

$$
D_{\mu} X^{M}:=\partial_{\mu} X^{M}-A_{\mu}^{i} \partial_{i} X^{M}
$$

The Nambu $\hat{d}$-bracket (1.7) is defined here, simply without a local factor, by 1

$$
\left\{Y_{1}, Y_{2}, \cdots, Y_{\hat{d}}\right\}_{\mathrm{N} . \mathrm{B}}:=\epsilon^{i_{1} i_{2} \cdots i_{\hat{d}}} \partial_{i_{1}} Y_{1} \partial_{i_{2}} Y_{2} \cdots \partial_{i_{\hat{d}}} Y_{\hat{d}}
$$

Integrating out all the auxiliary fields, using their on-shell values, the action reduces to the Nambu-Goto action, $S_{\text {New }} \equiv S_{\text {N.G. }}$, and hence the classical equivalence. The novelty of the above reformulation was the appearance of the gauge interaction and the Nambu bracket squared potential. The latter basically stems from an identity rewriting the determinant as the Nambu bracket squared:

$$
\operatorname{det}\left(\partial_{i} X^{M} \partial_{j} X_{M}\right)=\frac{1}{\hat{d} !}\left\{X^{M_{1}}, X^{M_{2}}, \cdot \cdot, X^{M_{\hat{d}}}\right\}_{\mathrm{N} . \mathrm{B}}\left\{X_{M_{1}}, X_{M_{2}}, \cdot \cdot, X_{M_{\hat{d}}}\right\}_{\mathrm{N} . \mathrm{B}} \cdot
$$

A physical picture behind the reformulation is to describe a single brane as a condensation of multiple lower-dimensional branes, i.e. a $p$-brane by $(d-1)$-branes: IKKT matrix model [3] is a multiple D-instanton description of type IIB superstring, while BFSS matrix model [4] is a multiple D0-brane description of supermembrane (see also [28]) . Obviously, the choice of $\hat{d}=0$ and $d=p+1$ corresponds to the wellknown "Polyakov" action which was actually first conceived by Brink, Di Vecchia, Howe, Tucker [29, 30]. On the other hand, with a gauge fixing for $\omega$ to be constant, the other extreme choice of $d=0, \hat{d}=p+1$ leads to the Schild action [31]. Furthermore, the association of the digits, 2 and 3 to string and $\mathcal{M}$-theory become manifest within the reformulation: For example, the fact that the codimension of D-branes is 2 suggests to choose $\hat{d}=2$, which leads to the two-algebra as in Yang-Mills. Likely the choice of $p=5$, $d=3, \hat{d}=3$ suggests that the Bagger-Lambert-Gustavsson model with an infinite dimensional gauge group describes a M5-brane as a condensation of multiple M2-branes.

The reformulation of the Nambu-Goto action (1.13) is purely bosonic. In order to establish a firm connection to string/ $\mathcal{M}$-theory one needs to supersymmetrize them. The requirement of supersymmetry may give rise to a constraint on the $a$ priori arbitrary decomposition, $p+1=d+\hat{d}$.

Our main interest is to supersymmetrize the action (1.13). For $d=1$ case, supersymmetric actions are ready to be read-off from an earlier work by Bergshoeff, Sezgin, Tanii and Townsend [37]. In its appendix the authors listed light-cone gauge fixed supersymmetric actions for various $p$-branes in diverse

\footnotetext{
${ }^{1}$ As usual, $\epsilon^{i_{1} i_{2} \cdots i} \hat{d}$ is the totally anti-symmetric $\hat{d}$-dimensional tensor of the normalization $\epsilon^{12 \cdots \hat{d}}=1$.
} 
spacetime dimensions. Utilizing the identity (1.16), in terms of Nambu $p$-bracket, their light-cone gauge fixed supersymmetric $p$-brane actions can be reexpressed in a compact form:

$\mathcal{L}_{\text {L.C. }}=\frac{1}{2}\left(D_{t} X^{I}\right)^{2}-\frac{1}{2 p !}\left\{X^{I_{1}}, X^{I_{2}}, \cdot \cdot, X^{I_{p}}\right\}_{\mathrm{N} . \mathrm{B}}^{2}+i \frac{1}{2} \bar{\Psi} D_{t} \Psi+\frac{1}{2(p-1) !} \bar{\Psi} \Gamma^{I_{1} I_{2} \cdots I_{p-1}}\left\{X_{I_{1}}, \cdots, X_{I_{p-1}}, \Psi\right\}_{\mathrm{N} . \mathrm{B}}$.

As usual, the Fierz identity required for the supersymmetry invariance, restricts the possible values of $p$ and the spacetime dimension $D$ (as for $I=1,2, \cdots, D-2$ ):

$$
\begin{array}{ll}
p=1: & D=3,4,6,10 \\
p=2: & D=4,5,7,11 \\
p=3: & D=6,8 \\
p=4: & D=9 \\
p=5: & D=10 .
\end{array}
$$

In the present paper, we consider an alternative choice of $d=0$. In particular, we focus on a supermembrane propagating in eleven-dimensional flat spacetime. As we take the choice of $\hat{d}=3$, the bosonic action is of Schild type and it will contain manifestly Nambu three-brackets.

The organization of the rest of the paper is as follows. In section 2 we present our main result: We construct a 3-algebra based action for a supermembrane in eleven dimensions. The action is invariant under global supersymmetry, local kappa-symmetry and worldvolume diffeomorphism. In section 3 we perform a double dimensional reduction and obtain a covariant, two-algebra based action for a type IIA superstring in ten dimensions. In a similar fashion, in section 4 we construct a covariant, two-algebra based action for a type IIB superstring. Section 5 contains our discussion and the appendix carries some useful identities.

Note added: While this work was being finished, two related papers, [35] and [36], appeared on arXiv. The former discusses a bosonic three-algebra squared action and the latter presents a supersymmetric version of it with the spacetime dimension four and the signature $2+2$. On the other hand, our action is for the supermembrane in eleven-dimensional Minkowskian spacetime. 


\section{Three-algebra based action for supermembrane in eleven dimensions}

\subsection{The action}

We propose the following action for the three-algebra description of a supermembrane in eleven dimensions:

$$
\begin{gathered}
\mathcal{S}_{\mathrm{M} 2}=\int \mathrm{d}^{3} \xi\left(\mathcal{L}_{\omega}+\mathcal{L}_{\mathrm{WZ}}\right), \\
\mathcal{L}_{\omega}=\frac{1}{12} \omega^{-1}\left\langle\Pi^{M}, \Pi^{N}, \Pi^{P}\right\rangle\left\langle\Pi_{M}, \Pi_{N}, \Pi_{P}\right\rangle-\frac{1}{2} \omega, \\
\mathcal{L}_{\mathrm{wZ}}=-i \frac{1}{2} \epsilon^{i j k} \bar{\theta} \Gamma_{M N} \partial_{i} \theta\left(\Pi_{j}^{M} \partial_{k} X^{N}-\frac{1}{3} \bar{\theta} \Gamma^{M} \partial_{j} \theta \bar{\theta} \Gamma^{N} \partial_{k} \theta\right),
\end{gathered}
$$

which contains eleven-dimensional target spacetime coordinates $X^{M}$, a Majorana spinor $\theta$ and a scalar density field $\omega$. The former two are dynamical while the last one is auxiliary. With the supersymmetry invariant pull-back:

$$
\Pi_{i}^{M}:=\partial_{i} X^{M}-i \bar{\theta} \Gamma^{M} \partial_{i} \theta
$$

we set

$$
\left\langle\Pi^{L}, \Pi^{M}, \Pi^{N}\right\rangle:=\epsilon^{i j k} \Pi_{i}^{L} \Pi_{j}^{M} \Pi_{k}^{N},
$$

which has the following expansion in terms of the Nambu-bracket (1.15).2

$$
\begin{aligned}
\left\langle\Pi^{L}, \Pi^{M}, \Pi^{N}\right\rangle:= & \left\{X^{L}, X^{M}, X^{N}\right\}_{\mathrm{N} . \mathrm{B}}-3 i \bar{\theta} \Gamma^{[L}\left\{X^{M}, X^{N]}, \theta\right\}_{\mathrm{N} . \mathrm{B}}+3 \bar{\theta}\left\{\Gamma^{[L} \theta, X^{M}, \bar{\theta} \Gamma^{N]}\right\}_{\mathrm{N} . \mathrm{B}} \theta \\
& -i \bar{\theta}_{\alpha} \bar{\theta}_{\beta} \bar{\theta}_{\gamma}\left\{\left(\Gamma^{[L} \theta\right)^{\alpha},\left(\Gamma^{M} \theta\right)^{\beta},\left(\Gamma^{N]} \theta\right)^{\gamma}\right\}_{\mathrm{N} . \mathrm{B}} .
\end{aligned}
$$

Similarly, the Wess-Zumino part of the action can be also reexpressed in terms of the Nambu-bracket:

$$
\begin{aligned}
\mathcal{L}_{\mathrm{WZ}}= & -i \frac{1}{2} \bar{\theta} \Gamma_{M N}\left\{X^{M}, X^{N}, \theta\right\}_{\mathrm{N} . \mathrm{B}}+\frac{1}{2} \bar{\theta}_{\alpha} \bar{\theta}_{\beta}\left\{\left(\Gamma_{M N} \theta\right)^{\alpha},\left(\Gamma^{M} \theta\right)^{\beta}, X^{N}\right\}_{\mathrm{N} . \mathrm{B}} \\
& -i \frac{1}{6} \bar{\theta}_{\alpha} \bar{\theta}_{\beta} \bar{\theta}_{\gamma}\left\{\left(\Gamma_{M N} \theta\right)^{\alpha},\left(\Gamma^{M} \theta\right)^{\beta},\left(\Gamma^{N} \theta\right)^{\gamma}\right\}_{\mathrm{N} . \mathrm{B}} .
\end{aligned}
$$

Thus, all the derivatives appear only through Nambu three-brackets.

Let us now introduce a shorthand notation for the induced metric:

$$
g_{i j}:=\Pi_{i}^{M} \Pi_{M j},
$$

and denote its determinant by $g:=\operatorname{det}\left(g_{i j}\right)$ as usual.

All the equations of motion are then summarized by:

$$
\begin{aligned}
& \omega-\sqrt{-g} \equiv 0 \\
& g^{i j} \Pi_{i}^{M} \Gamma_{M}(1-\Gamma) \partial_{j} \theta \equiv 0, \\
& \partial_{i}\left(\sqrt{-g} g^{i j} \Pi_{j}^{M}\right)-i \epsilon^{i j k} \partial_{i} \bar{\theta} \Gamma^{M}{ }_{N} \partial_{j} \theta \Pi_{k}^{N} \equiv 0 .
\end{aligned}
$$

\footnotetext{
${ }^{2}$ Note that the bracket $[L, M, N]$ denotes the anti-symmetrization of the three indices with an overall factor $\frac{1}{6}$, i.e of 'strength one'.
} 
From an identity analogue to (1.16):

$$
\frac{1}{6}\left\langle\Pi^{M}, \Pi^{N}, \Pi^{P}\right\rangle\left\langle\Pi_{M}, \Pi_{N}, \Pi_{P}\right\rangle=\operatorname{det}\left(\Pi_{i}^{M} \Pi_{j M}\right),
$$

integrating out the auxiliary scalar assuming the on-shell value $\omega \equiv \sqrt{-g}$, our proposed action (2.1) reduces to the well-known supersymmetric Nambu-Goto action for M2-brane by Bergshoeff, Sezgin and Townsend [32,33]:

$$
\mathcal{S}_{\mathrm{M} 2} \equiv \int \mathrm{d}^{3} \xi\left[-\sqrt{-\operatorname{det}\left(\Pi_{i}^{M} \Pi_{j M}\right)}-i \frac{1}{2} \epsilon^{i j k} \bar{\theta} \Gamma_{M N} \partial_{i} \theta\left(\Pi_{j}^{M} \partial_{k} X^{N}-\frac{1}{3} \bar{\theta} \Gamma^{M} \partial_{j} \theta \bar{\theta} \Gamma^{N} \partial_{k} \theta\right)\right] .
$$

\subsection{Symmetries}

The action (2.1) is invariant under the following transformations.

- Target-spacetime supersymmetry:

$$
\delta_{\varepsilon} \theta=\varepsilon, \quad \delta_{\varepsilon} X^{M}=-i \bar{\theta} \Gamma^{M} \varepsilon, \quad \delta_{\varepsilon} \omega=0,
$$

which leaves $\Pi_{i}^{M}$ and $\mathcal{L}_{\omega}$ invariant, while transforms $\mathcal{L}_{\mathrm{wZ}}$ to a total derivative:

$$
\delta_{\varepsilon} \mathcal{L}_{\mathrm{WZ}}=\partial_{i}\left(\bar{\varepsilon} \eta^{i}\right)
$$

where $\varepsilon$ is a constant supersymmetry parameter and

$$
\begin{gathered}
\eta^{i}:=-i \frac{1}{2} \epsilon^{i j k}\left\{\Gamma_{M N} \theta \partial_{j} X^{M} \partial_{k} X^{N}-i\left(\Gamma_{M N} \partial_{j} \theta \bar{\theta} \Gamma^{M} \partial_{k} \theta-\Gamma^{M} \partial_{k} \theta \bar{\theta} \Gamma_{M N} \partial_{j} \theta\right) X^{N}\right. \\
\left.-\frac{1}{15}\left(\Gamma_{M N} \theta \bar{\theta} \Gamma^{M} \partial_{j} \theta+\Gamma^{M} \theta \bar{\theta} \Gamma_{M N} \partial_{j} \theta\right) \bar{\theta} \Gamma^{N} \partial_{k} \theta\right\}
\end{gathered}
$$

- Local 32-component fermionic symmetry:

$$
\begin{aligned}
& \delta_{\zeta} \theta=[1+(\omega / \sqrt{-g}) \Gamma] \zeta, \\
& \delta_{\zeta} X^{M}=i \bar{\theta} \Gamma^{M} \delta_{\zeta} \theta \\
& \delta_{\zeta} \omega=4 i \omega g^{-1 i j} \Pi_{i}^{M} \partial_{j} \bar{\theta} \Gamma_{M} \zeta,
\end{aligned}
$$

where $\zeta$ is an arbitrary local 32-component spinorial parameter and $\Gamma$ is as in $[32,33]$ :

$$
\Gamma:=\frac{1}{6 \sqrt{-g}} \Gamma_{L M N}\left\langle\Pi^{L}, \Pi^{M}, \Pi^{N}\right\rangle
$$

satisfying

$$
\Gamma^{2}=1
$$

Under the transformation above (2.14), the Lagrangian transforms to a total derivative:

$$
\delta_{\zeta}\left(\mathcal{L}_{\omega}+\mathcal{L}_{\mathrm{wZ}}\right)=\partial_{i}\left(\bar{\psi}^{i} \delta_{\zeta} \theta\right)
$$


where

$\bar{\psi}^{i}:=-\frac{1}{2} \epsilon^{i j k}\left[i \partial_{j} X^{M} \partial_{k} X^{N} \bar{\theta} \Gamma_{M N}+\left(\bar{\theta} \Gamma^{M} \partial_{j} \theta \bar{\theta} \Gamma_{M N}+\bar{\theta} \Gamma_{M N} \partial_{j} \theta \bar{\theta} \Gamma^{M}\right)\left(\partial_{k} X^{N}-i \frac{1}{3} \bar{\theta} \Gamma^{N} \partial_{k} \theta\right)\right]$

In particular, taking the choice $\zeta=(1+\omega / \sqrt{-g})^{-1}(1+\Gamma) \kappa$ leads to a symmetry:

$$
\begin{aligned}
& \delta_{\kappa} \theta=(1+\Gamma) \kappa \\
& \delta_{\kappa} X^{M}=i \bar{\theta} \Gamma^{M} \delta_{\kappa} \theta, \\
& \delta_{\kappa} \omega=4 i \frac{\omega \sqrt{-g}}{\omega+\sqrt{-g}} g^{-1 i j} \Pi_{i}^{N} \partial_{j} \bar{\theta} \Gamma_{N} \delta_{\kappa} \theta,
\end{aligned}
$$

where $\kappa$ is an arbitrary local fermionic parameter so that the transformations of $\theta$ and $X^{M}$ coincide with the kappa-symmetry in $[32,33]$.

On the other hand, an alternative, in fact complimentary, choice $\zeta=(1+\omega / \sqrt{-g})^{-1}[1-(\omega / \sqrt{-g}) \Gamma] \kappa^{\prime}$ leads to a symmetry:

$$
\begin{aligned}
& \delta_{\kappa^{\prime}} \theta=(1-\omega / \sqrt{-g}) \kappa^{\prime}, \\
& \delta_{\kappa^{\prime}} X^{M}=i \bar{\theta} \Gamma^{M} \delta_{\kappa^{\prime}} \theta, \\
& \delta_{\kappa^{\prime}} \omega=4 i \frac{\omega \sqrt{-g}}{\omega+\sqrt{-g}} g^{-1 i j} \Pi_{i}^{N} \partial_{j} \bar{\theta} \Gamma_{N}[1-(\omega / \sqrt{-g}) \Gamma] \kappa^{\prime} .
\end{aligned}
$$

On-shell (2.8), these transformations are trivial and hence cannot be used to reduce the fermionic physical degrees further after a $\kappa$-gauge fixing. More discussion on trivial symmetry transformations we refer e.g. [38]. Combining (2.19) and (2.20) gives back the generic transformation (2.14), and hence the former two are complimentary to each other.

- Worldvolume diffeomorphism:

$$
\delta_{v} X^{M}=v^{i} \partial_{i} X^{M}, \quad \delta_{v} \theta=v^{i} \partial_{i} \theta, \quad \delta_{v} \omega=\partial_{i}\left(\omega v^{i}\right),
$$

where $v^{i}=\delta \xi^{i}$ is an arbitrary local bosonic parameter, and the Lagrangian transforms to a total derivative as

$$
\delta_{v} \mathcal{L}_{\omega}=\partial_{i}\left(v^{i} \mathcal{L}_{\omega}\right), \quad \delta_{v} \mathcal{L}_{\mathrm{WZ}}=\partial_{i}\left(v^{i} \mathcal{L}_{\mathrm{WZ}}\right)
$$




\section{Double dimensional reduction to Type IIA superstring theory}

Double dimensional reduction [34] of our supermembrane action (2.1), putting $\xi^{2} \equiv X^{10}, \Gamma^{(11)}:=\Gamma^{10}$, straightforwardly leads to the following reformulation of the type IIA superstring action by Green and Schwarz $[39,40], 3$

$$
\mathcal{S}_{\mathrm{IIA}}=\int \mathrm{d}^{2} \xi\left(\mathcal{L}_{\omega}+\mathcal{L}_{\mathrm{WZ}}\right)
$$

where with $i=1,2, M=0,1, \cdots, 9$,

$$
\begin{aligned}
\mathcal{L}_{\omega} & =\frac{1}{4} \omega^{-1}\left\langle\Pi^{M}, \Pi^{N}\right\rangle\left\langle\Pi_{M}, \Pi_{N}\right\rangle-\frac{1}{2} \omega, \\
\mathcal{L}_{\mathrm{WZ}} & =i \epsilon^{i j} \partial_{i} X^{M} \bar{\theta} \Gamma_{M(11)} \partial_{j} \theta-\frac{1}{2} \epsilon^{i j} \bar{\theta} \Gamma_{M(11)} \partial_{i} \theta \bar{\theta} \Gamma^{M} \partial_{j} \theta,
\end{aligned}
$$

and

$$
\Pi_{i}^{M}=\partial_{i} X^{M}-i \bar{\theta} \Gamma^{M} \partial_{i} \theta, \quad\left\langle\Pi^{M}, \Pi^{N}\right\rangle:=\epsilon^{i j} \Pi_{i}^{M} \Pi_{j}^{N} .
$$

Note that $\theta$ is a ten-dimensional Majorana spinor which can decompose into a chiral and an anti-chiral Majorana spinor, and hence type IIA Majorana-Weyl spinors of opposite chiralities.

In terms of Nambu 2-bracket or Poisson bracket, we can write

$$
\begin{aligned}
& \left\langle\Pi^{M}, \Pi^{N}\right\rangle=\left\{X^{M}, X^{N}\right\}_{\text {P.B }}-2 i \bar{\theta}\left\{X^{[M}, \Gamma^{N]} \theta\right\}_{\text {P.B }}+\bar{\theta}_{\alpha} \bar{\theta}_{\beta}\left\{\left(\Gamma^{[M} \theta\right)^{\alpha},\left(\Gamma^{N]} \theta\right)^{\beta}\right\}_{\text {P.B }}, \\
& \mathcal{L}_{\mathrm{WZ}}=i \bar{\theta}\left\{X^{M}, \Gamma_{M(11)} \theta\right\}_{\text {P.B }}+\frac{1}{2} \bar{\theta}_{\alpha} \bar{\theta}_{\beta}\left\{\left(\Gamma_{M(11)} \theta\right)^{\alpha},\left(\Gamma^{M} \theta\right)^{\beta}\right\}_{\text {P.B }} .
\end{aligned}
$$

Thus, all the derivatives appear only through Poisson brackets.

Integrating out the auxiliary scalar field $\omega$, our action (3.1) reduces to the Green-Schwarz action for type IIA superstring [39, 40].

The action (3.1) is invariant under the following transformations.

- Target-spacetime supersymmetry:

$$
\delta_{\varepsilon} \theta=\varepsilon, \quad \delta_{\varepsilon} X^{M}=-i \bar{\theta} \Gamma^{M} \varepsilon, \quad \delta_{\varepsilon} \omega=0,
$$

which leaves $\mathcal{L}_{\omega}$ invariant and transforms $\mathcal{L}_{\mathrm{WZ}}$ to a total derivative:

$$
\delta_{\varepsilon} \mathcal{L}_{\mathrm{WZ}}=\partial_{i}\left(\bar{\varepsilon} \eta_{\mathrm{IIA}}^{i}\right)
$$

where $\varepsilon$ is a constant supersymmetry parameter and

$$
\eta_{\mathrm{IIA}}^{i}:=\epsilon^{i j}\left[-i \Gamma_{M(11)} \theta \partial_{j} X^{M}-\frac{1}{6} \Gamma_{M(11)} \theta \bar{\theta} \Gamma^{M} \partial_{j} \theta-\frac{1}{6} \Gamma^{M} \theta \bar{\theta} \Gamma_{M(11)} \partial_{j} \theta\right] .
$$

\footnotetext{
${ }^{3}$ For the dimensional reduction of BLG model see e.g. [41, 42].
} 
- Local 32-component fermionic symmetry:

$$
\begin{aligned}
& \delta_{\zeta} \theta=\left[1+(\omega / \sqrt{-g}) \tilde{\Gamma} \Gamma^{(11)}\right] \zeta, \\
& \delta_{\zeta} X^{M}=i \bar{\theta} \Gamma^{M} \delta_{\zeta} \theta, \\
& \delta_{\zeta} \omega=4 i \omega g^{-1 i j} \Pi_{i}^{M} \partial_{j} \bar{\theta} \Gamma_{M} \zeta,
\end{aligned}
$$

where $\zeta$ is an arbitrary 32-component local fermionic parameter and

$$
\tilde{\Gamma}:=\frac{1}{2 \sqrt{-g}} \epsilon^{i j} \Pi_{i}^{M} \Pi_{j}^{N} \Gamma_{M N}
$$

satisfying $\tilde{\Gamma}^{2}=1$. Under the transformation $(3.8)$ the Lagrangian transforms to a total derivative:

$$
\delta_{\zeta}\left(\mathcal{L}_{\omega}+\mathcal{L}_{\mathrm{WZ}}\right)=\partial_{i}\left(\bar{\psi}_{\mathrm{IIA}}^{i} \delta_{\zeta} \theta\right)
$$

where

$$
\bar{\psi}_{\mathrm{IIA}}^{i}:=\epsilon^{i j}\left(-i \partial_{j} X^{M} \bar{\theta} \Gamma_{M(11)}-\frac{1}{2} \bar{\theta} \Gamma_{M(11)} \partial_{j} \theta \bar{\theta} \Gamma^{M}-\frac{1}{2} \bar{\theta} \Gamma^{M} \partial_{j} \bar{\theta} \Gamma_{M(11)}\right) .
$$

As in the case of the three-algebra based supermembrane action (2.19), (2.20), the local fermionic symmetry consists of $\kappa$-symmetry and trivial transformation.

- Worldvolume diffeomorphism:

$$
\delta_{v} X^{M}=v^{i} \partial_{i} X^{M}, \quad \delta_{v} \theta=v^{i} \partial_{i} \theta, \quad \delta_{v} \omega=\partial_{i}\left(\omega v^{i}\right)
$$

The Lagrangian transforms to a total derivative as (2.22).

\section{Type IIB superstring theory and IKKT matrix model}

In a similar fashion to our type IIA superstring action (3.1), the Schild version of type IIB superstring action assumes the form:

$$
\mathcal{S}_{\mathrm{IIB}}=\int \mathrm{d}^{2} \xi\left(\mathcal{L}_{\omega}+\mathcal{L}_{\mathrm{WZ}}\right)
$$

where

$$
\begin{aligned}
\mathcal{L}_{\omega} & =\frac{1}{4} \omega^{-1}\left\langle\Pi^{M}, \Pi^{N}\right\rangle\left\langle\Pi_{M}, \Pi_{N}\right\rangle-\frac{1}{2} \omega \\
\mathcal{L}_{\mathrm{wZ}} & =-i \epsilon^{i j} \partial_{i} X^{M}\left(\bar{\theta}^{1} \Gamma_{M} \partial_{j} \theta^{1}-\bar{\theta}^{2} \Gamma_{M} \partial_{j} \theta^{2}\right)+\epsilon^{i j} \bar{\theta}^{1} \Gamma^{M} \partial_{i} \theta^{1} \bar{\theta}^{2} \Gamma_{M} \partial_{j} \theta^{2} \\
& =-i \bar{\theta}^{1} \Gamma_{M}\left\{X^{M}, \theta^{1}\right\}_{\text {P.B }}+i \bar{\theta}^{2} \Gamma_{M}\left\{X^{M}, \theta^{2}\right\}_{\text {P.B }}-\bar{\theta}_{\alpha}^{1} \bar{\theta}_{\beta}^{2}\left\{\left(\Gamma_{M} \theta^{1}\right)^{\alpha},\left(\Gamma^{M} \theta^{2}\right)^{\beta}\right\}_{\text {P.B }} .
\end{aligned}
$$

With a pair of Majorana-Weyl spinors of a same chirality,

$$
\Gamma^{(11)} \theta^{1}=\theta^{1}, \quad \Gamma^{(11)} \theta^{2}=\theta^{2},
$$


the supersymmetry invariant pull-back is given by

$$
\Pi_{i}^{M}=\partial_{i} X^{M}-i\left(\bar{\theta}^{1} \Gamma^{M} \partial_{i} \theta^{1}+\bar{\theta}^{2} \Gamma^{M} \partial_{i} \theta^{2}\right) .
$$

Our type IIB superstring action (4.1) is invariant under the following transformations.

- Target-spacetime supersymmetry:

$$
\delta_{\varepsilon} \theta^{1}=\varepsilon^{1}, \quad \delta_{\varepsilon} \theta^{2}=\varepsilon^{2}, \quad \delta_{\varepsilon} X^{M}=-i \bar{\theta}^{1} \Gamma^{M} \varepsilon^{1}-i \bar{\theta}^{2} \Gamma^{M} \varepsilon^{2}, \quad \delta_{\varepsilon} \omega=0,
$$

which leaves $\mathcal{L}_{\omega}$ invariant and transforms $\mathcal{L}_{\mathrm{WZ}}$ to a total derivative:

$$
\delta_{\varepsilon} \mathcal{L}_{\mathrm{WZ}}=\partial_{i}\left(\bar{\varepsilon}^{1} \eta_{\mathrm{IIB}}^{1 i}+\bar{\varepsilon}^{2} \eta_{\mathrm{IIB}}^{2 i}\right)
$$

where $\varepsilon^{1}, \varepsilon^{2}$ are constant supersymmetry parameters and

$$
\begin{aligned}
& \eta_{\mathrm{IIB}}^{1 i}:=\epsilon^{i j}\left(i \partial_{j} X^{M} \Gamma_{M} \theta^{1}+\frac{1}{3} \Gamma^{M} \theta^{1} \bar{\theta}^{1} \Gamma_{M} \partial_{j} \theta^{1}\right) \\
& \eta_{\mathrm{IIB}}^{2 i}:=-\epsilon^{i j}\left(i \partial_{j} X^{M} \Gamma_{M} \theta^{2}+\frac{1}{3} \Gamma^{M} \theta^{2} \bar{\theta}^{2} \Gamma_{M} \partial_{j} \theta^{2}\right) .
\end{aligned}
$$

- Local 32-component fermionic symmetry:

$$
\begin{aligned}
& \delta_{\zeta} \theta^{1}=\left(1+\frac{\omega}{\sqrt{-g}} \tilde{\Gamma}\right) \zeta^{1}, \\
& \delta_{\zeta} \theta^{2}=\left(1-\frac{\omega}{\sqrt{-g}} \tilde{\Gamma}\right) \zeta^{2}, \\
& \delta_{\zeta} X^{M}=i \bar{\theta}^{1} \Gamma^{M} \delta_{\zeta} \theta^{1}+i \bar{\theta}^{2} \Gamma^{M} \delta_{\zeta} \theta^{2}, \\
& \delta_{\zeta} \omega=4 i \omega g^{-1 i j} \Pi_{i}^{N}\left(\partial_{j} \bar{\theta}^{1} \Gamma_{N} \zeta^{1}+\partial_{j} \bar{\theta}^{2} \Gamma_{N} \zeta^{2}\right),
\end{aligned}
$$

where $\zeta$ is an arbitrary local fermionic parameter. The Lagrangian transforms to a total derivative,

$$
\delta_{\zeta}\left(\mathcal{L}_{\omega}+\mathcal{L}_{\mathrm{WZ}}\right)=\partial_{i}\left(\bar{\psi}_{\mathrm{IIB}}^{1 i} \delta_{\zeta} \theta^{1}+\bar{\psi}_{\mathrm{IIB}}^{2 i} \delta_{\zeta} \theta^{2}\right)
$$

where

$$
\begin{aligned}
& \bar{\psi}_{\mathrm{IIB}}^{1 i}:=\epsilon^{i j}\left(i \Pi_{j}^{\mu} \bar{\theta}^{1} \Gamma_{M}-\bar{\theta}^{2} \Gamma^{M} \partial_{j} \theta^{2} \bar{\theta}^{1} \Gamma_{M}\right), \\
& \bar{\psi}_{\mathrm{IIB}}^{2 i}:=-\epsilon^{i j}\left(i \Pi_{j}^{\mu} \bar{\theta}^{2} \Gamma_{M}-\bar{\theta}^{1} \Gamma^{M} \partial_{j} \theta^{1} \bar{\theta}^{2} \Gamma_{M}\right) .
\end{aligned}
$$

The local fermionic symmetry above consists of $\kappa$-symmetry and trivial transformation, as in the supermembrane and type IIA superstring cases.

- Worldvolume diffeomorphism:

$$
\delta_{v} X^{M}=v^{i} \partial_{i} X^{M}, \quad \delta_{v} \theta^{1}=v^{i} \partial_{i} \theta^{1}, \quad \delta_{v} \theta^{2}=v^{i} \partial_{i} \theta^{2}, \quad \delta_{v} \omega=\partial_{i}\left(\omega v^{i}\right),
$$


where $v^{i}=\delta \xi^{i}$ is an arbitrary local bosonic parameter. The Lagrangian transforms to a total derivative as (2.22).

Now, replacing $\theta^{2}$ by $i \theta^{2}$ through an analytic continuation [3], fixing the gauge $\theta^{1} \equiv \theta^{2}$ through the local fermionic symmetry and $\omega \equiv 1$ through the diffeomorphism, the action reduces to the form:

$$
\mathcal{S}_{\text {IIB }} \equiv \int \mathrm{d}^{2} \xi \frac{1}{4}\left\{X^{M}, X^{N}\right\}_{\text {P.B }}\left\{X_{M}, X_{N}\right\}_{\text {P.B }}-2 i \bar{\theta}^{1} \Gamma_{M}\left\{X^{M}, \theta^{1}\right\}_{\text {P.B }},
$$

which straightforwardly leads, after a matrix regularization of the Poisson bracket, to the type IIB IKKT matrix model [3]. Thus, our two-algebra based covariant type IIB superstring action (4.1) presents a direct derivation of the IKKT matrix model starting from the covariant superstring action (4.1) 4

\section{Discussion}

In summary, we have constructed covariant actions for type IIA, type IIB superstrings in ten dimensions, and supermembrane in eleven dimensions, which are invariant under global supersymmetry, local fermionic symmetry and worldvolume diffeomorphism. All the derivatives therein appear through Nambu brackets such that the two-algebra structure of superstring theory and the three-algebra structure of $\mathcal{M}$-theory become manifest. Nambu two and three brackets naturally arise since the dimensions of the string worldsheet and the membrane worldvolume are two and three respectively. One advantage to employ the Nambu brackets is the simplicity of the double dimensional reduction: The three-bracket clearly reduces to the two-bracket.

Since our resulting actions (2.1), (3.1), (4.1) contain higher than second order terms, the generalization of the inner product of Filippov $n$-Lie algebra as in (1.8), (1.9) is necessary. Like the type IIB case, suitable gauge fixing to simplify the actions for supermembrane and type IIA superstring is desired.

The BFSS matrix model $[4,5]$ is a light-cone gauge fixed action for supermembrane such that it describes only the sector of classically fixed light-cone momentum. Our covariant supermembrane action (2.1) is classically equivalent to the BFSS matrix model. However, the quantum equivalence is to be investigated in future work.

\section{Acknowledgments}

We wish to thank Kazuyuki Furuuchi, Seungjoon Hyun, Corneliu Sochichiu for useful comments, and especially Johanna Erdmenger at Max-Planck Institut fur Physik, Munchen for helpful discussion as well as hospitality during our visit. The work is in part supported by the Korea Foundation for International Cooperation of Science \& Technology with grant number K20821000003-08B1200-00310, by the Center for Quantum Spacetime of Sogang University with grant number R11-2005-021, by the Korea Science and Engineering Foundation with grant number R01-2007-000-20062-0, and by the Basic Research Program of the Korea Science and Engineering Foundation with grant number R01-2004-000-10651-0.

\footnotetext{
${ }^{4}$ Note that in the original derivation of the type IIB matrix model [3], the auxiliary scalar field vanished away during the matrix regularization as it is absorbed into either the trace or the matrix commutator, while in our scheme it is gauge fixed to be a constant.
} 


\section{A Useful Fierz identities in eleven dimensions}

In Minkowskian eleven dimensions, with an anti-symmetric charge conjugation matrix $C$, the gamma matrices satisfy

$$
\left(C \Gamma^{M_{1} M_{2} \cdots M_{n}}\right)^{T}=(-1)^{1+\frac{1}{2} n(n+1)} C \Gamma^{M_{1} M_{2} \cdots M_{n}},
$$

and a Fierz identity:

$$
\left(C \Gamma^{M}\right)_{(\alpha \beta}\left(C \Gamma_{M N}\right)_{\gamma \delta)}=0 .
$$

Thanks to this Fierz identity, various identities follow which are crucial for the supersymmetry and the local fermionic symmetry of the action 2.1 :

$$
\begin{gathered}
\epsilon^{i j k}\left(\bar{\varepsilon} \Gamma_{M N} \theta \partial_{j} \bar{\theta} \Gamma^{M} \partial_{k} \theta-2 \bar{\theta} \Gamma_{M N} \partial_{j} \theta \partial_{k} \bar{\theta} \Gamma^{M} \varepsilon+\partial_{j} \bar{\theta} \Gamma_{M N} \partial_{k} \theta \bar{\varepsilon} \Gamma^{M} \theta-2 \bar{\varepsilon} \Gamma_{M N} \partial_{j} \theta \bar{\theta} \Gamma^{M} \partial_{k} \theta\right)=0, \\
\epsilon^{i j k}\left(\bar{\varepsilon} \Gamma_{M N} \partial_{i} \theta \partial_{j} \bar{\theta} \Gamma^{M} \partial_{k} \theta+\partial_{i} \bar{\theta} \Gamma_{M N} \partial_{j} \theta \bar{\varepsilon} \Gamma^{M} \partial_{k} \theta\right)=0, \\
\epsilon^{i j k}\left(\bar{\varepsilon} \Gamma_{M N} \partial_{i} \theta \bar{\theta} \Gamma^{M} \partial_{j} \theta+\bar{\theta} \Gamma_{M N} \partial_{i} \theta \partial_{j} \bar{\theta} \Gamma^{M} \varepsilon\right) \bar{\theta} \Gamma^{N} \partial_{k} \theta \\
=\frac{1}{5} \epsilon^{i j k} \partial_{i}\left\{\left(\bar{\varepsilon} \Gamma_{M N} \theta \bar{\theta} \Gamma^{M} \partial_{j} \theta+\bar{\theta} \Gamma_{M N} \partial_{j} \theta \bar{\varepsilon} \Gamma^{M} \theta\right) \bar{\theta} \Gamma^{N} \partial_{k} \theta\right\} .
\end{gathered}
$$

Other useful identities include

$$
\begin{aligned}
\left(\Gamma^{M}\right)_{\gamma}^{[\alpha}\left(\Gamma_{M}\right)_{\delta}^{\beta]}= & \frac{11}{32} C^{-1 \alpha \beta} C_{\gamma \delta}+\frac{5}{32 \times 3 !}\left(\Gamma^{L M N} C^{-1}\right)^{\alpha \beta}\left(\Gamma_{L M N} C\right)_{\gamma \delta} \\
& +\frac{3}{32 \times 4 !}\left(\Gamma^{L M N P} C^{-1}\right)^{\alpha \beta}\left(\Gamma_{L M N P} C\right)_{\gamma \delta}, \\
\left(\Gamma^{M N}\right)_{(\gamma}^{[\alpha}\left(\Gamma_{N}\right)_{\delta)}^{\beta]}= & \frac{5}{16} C^{-1 \alpha \beta}\left(C \Gamma^{M}\right)_{\gamma \delta}+\frac{3}{32}\left(\Gamma^{M L_{1} L_{2}} C^{-1}\right)^{\alpha \beta}\left(C \Gamma_{L_{1} L_{2}}\right)_{\gamma \delta} \\
& +\frac{1}{16 \times 4 !}\left(\Gamma_{L_{1} L_{2} L_{3} L_{4}} C^{-1}\right)^{\alpha \beta}\left(C \Gamma^{M L_{1} L_{2} L_{3} L_{4}}\right)_{\gamma \delta}
\end{aligned}
$$

and

$$
\sqrt{-g} g^{-1 i j} \Pi_{j}^{M} \Gamma_{M} \Gamma=\sqrt{-g} g^{-1 i j} \Pi_{j}^{M} \Gamma \Gamma_{M}=\frac{1}{2} \epsilon^{i j k} \Pi_{j}^{L} \Pi_{k}^{M} \Gamma_{L M} .
$$




\section{References}

[1] J. Bagger and N. Lambert, Phys. Rev. D 75 (2007) 045020 [arXiv:hep-th/0611108];

J. Bagger and N. Lambert, Phys. Rev. D 77 (2008) 065008 [arXiv:0711.0955[hep-th]];

J. Bagger and N. Lambert, JHEP 0802 (2008) 105 [arXiv:0712.3738 [hep-th]].

[2] A. Gustavsson, Nucl. Phys. B 811 (2009) 66 [arXiv:0709.1260 [hep-th]].

[3] N. Ishibashi, H. Kawai, Y. Kitazawa and A. Tsuchiya, Nucl. Phys. B 498 (1997) 467 [arXiv:hep-th/9612115].

[4] T. Banks, W. Fischler, S. H. Shenker and L. Susskind, Phys. Rev. D 55 (1997) 5112 arXiv:hep-th/9610043].

[5] B. de Wit, J. Hoppe and H. Nicolai, Nucl. Phys. B 305 (1988) 545.

[6] J. Polchinski, "String theory. Vol. 2: Superstring theory and beyond" Cambridge, UK: Univ. Pr. (1998) $531 p$

[7] R. C. Myers, JHEP 9912 (1999) 022 [arXiv:hep-th/9910053].

[8] J. Gomis, A. J. Salim and F. Passerini, JHEP 0808 (2008) 002 [arXiv:0804.2186 [hep-th]].

[9] P. M. Ho and Y. Matsuo, JHEP 0806, 105 (2008) [arXiv:0804.3629 [hep-th]].

[10] P. M. Ho, Y. Imamura, Y. Matsuo and S. Shiba, JHEP 0808, 014 (2008) [arXiv:0805.2898 [hep-th]].

[11] C. Krishnan and C. Maccaferri, JHEP 0807, 005 (2008) [arXiv:0805.3125 [hep-th]].

[12] I. Jeon, J. Kim, N. Kim, S. W. Kim and J.-H. Park, JHEP 0807 (2008) 056 [arXiv:0805.3236 [hep-th]].

[13] J.-H. Park and C. Sochichiu, arXiv:0806.0335 [hep-th].

[14] I. A. Bandos and P. K. Townsend, arXiv:0806.4777 [hep-th].

[15] G. Bonelli, A. Tanzini and M. Zabzine, arXiv:0807.5113 [hep-th].

[16] I. A. Bandos and P. K. Townsend, JHEP 0902 (2009) 013 [arXiv:0808.1583 [hep-th]].

[17] I. Jeon, J. Kim, N. Kim, B. H. Lee and J.-H. Park, arXiv:0809.0856 [hep-th].

[18] K. Lee, S. Lee and J.-H. Park, JHEP 0811 (2008) 014 [arXiv:0809.2924 [hep-th]].

[19] M. A. Ganjali, arXiv:0811.2976 [hep-th].

[20] M. A. Ganjali, arXiv:0901.2642 [hep-th].

[21] V. T. Filippov, “n-Lie algebras,” Sib. Mat. Zh., 26, No 6, 126-140 (1985). 
[22] Y. Nambu, Phys. Rev. D 72405 (1973).

[23] G. Papadopoulos, JHEP 0805 (2008) 054 [arXiv:0804.2662 [hep-th]].

[24] J. P. Gauntlett and J. B. Gutowski, arXiv:0804.3078 [hep-th].

[25] M. Axenides and E. Floratos, JHEP 0703 (2007) 093 [arXiv:hep-th/0608017].

[26] M. Axenides and E. Floratos, arXiv:0809.3493 [hep-th].

[27] Y. Nambu, Lectures at the Copenhagen symposium, 1970; T. Goto, Prog. Theor. Phys. 46 (1971) 1560.

[28] H. S. Yang, I. Kim and B. H. Lee, Phys. Rev. D 58 (1998) 085018 [arXiv:hep-th/9806112].

[29] L. Brink, P. Di Vecchia and P. S. Howe, Phys. Lett. B 65 (1976) 471.

[30] P. S. Howe and R. W. Tucker, J. Phys. A 10 (1977) L155.

[31] A. Schild, Phys. Rev. D 16 (1977) 1722.

[32] E. Bergshoeff, E. Sezgin and P. K. Townsend, Phys. Lett. B 189 (1987) 75.

[33] E. Bergshoeff, E. Sezgin and P. K. Townsend, Annals Phys. 185 (1988) 330.

[34] M. J. Duff, P. S. Howe, T. Inami and K. S. Stelle, Phys. Lett. B 191 (1987) 70.

[35] M. Sato, arXiv:0902.1333 [hep-th].

[36] K. Furuuchi and D. Tomino, arXiv:0902.2041 [hep-th].

[37] E. Bergshoeff, E. Sezgin, Y. Tanii and P. K. Townsend, Annals Phys. 199 (1990) 340.

[38] M. Henneaux and C. Teitelboim, Quantization of gauge systems (Princeton University Press, 1992).

[39] M. B. Green and J. H. Schwarz, Phys. Lett. B 136 (1984) 367.

[40] M. B. Green and J. H. Schwarz, Nucl. Phys. B 243 (1984) 285.

[41] M. A. Santos and I. V. Vancea, arXiv:0809.0256 [hep-th].

[42] P. Franche, arXiv:0811.1443 [hep-th]. 